\title{
THE STATUS OF THE CMS EXPERIMENT
}

\author{
DAN GREEN \\ Fermilab
}

\begin{abstract}
The CMS experiment was completely assembled in the fall of 2008 after a decade of design, construction and installation. During the last two years, cosmic ray data were taken on a regular basis. These data have enabled CMS to align the detector components, both spatially and temporally. Initial use of muons has also established the relative alignment of the CMS tracking and muon systems. In addition, the CMS calorimetry has been crosschecked with test beam data, thus providing an initial energy calibration of CMS calorimetry to about $5 \%$.

The CMS magnet has been powered and field mapped. The trigger and data acquisition systems have been installed and run at full speed. The tiered data analysis system has been exercised at full design bandwidth for Tier0, Tier1 and Tier2 sites. Monte Carlo simulation of the CMS detector has been constructed at a detailed geometric level and has been tuned to test beam and other production data to provide a realistic model of the CMS detector prior to first collisions.
\end{abstract}

\section{The Surface Assembly Hall - Magnet Test and Cosmic Rays}

A schematic view of CMS is shown in Fig.1. The main design features are a large solenoid with a 4T field in which are mounted an all silicon tracking system with pixels and strips, a crystal electromagnetic calorimeter and a brass/scintillator hadronic calorimeter. Outside the magnet the flux return yoke is instrumented with muon chambers.

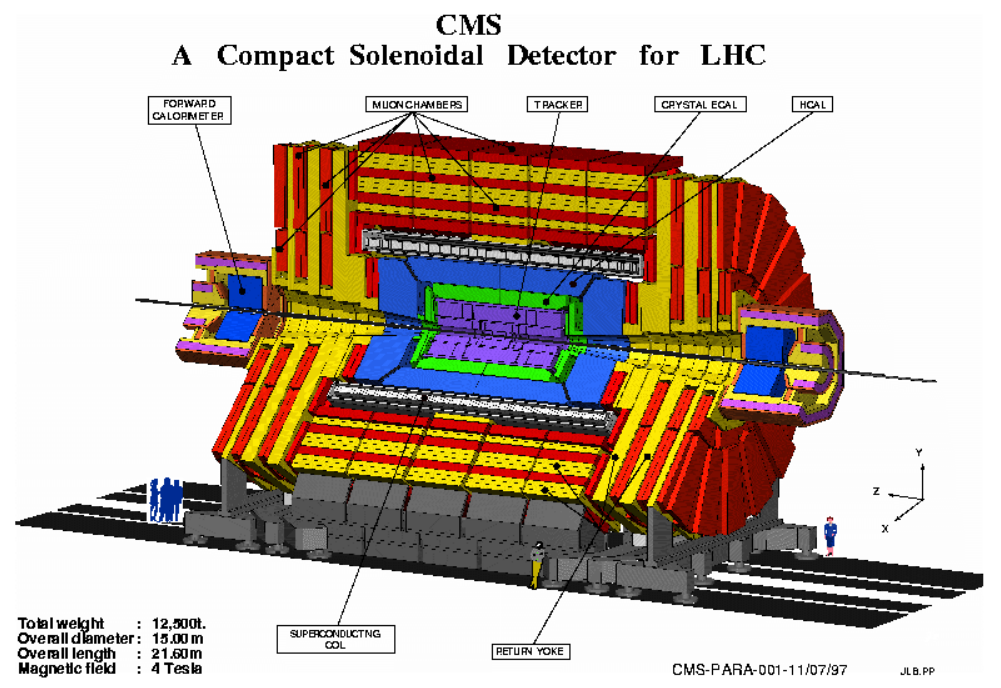

Figure 1: A schematic view of the CMS detector. The main subsystems are labeled; the tracker, ECAL, HCAL, and muon systems, MB/ME. Calorimetry covers the central region, EB. HB, the endcap region, EE, HE and the forward region, HF. 
The CMS detector was explicitly designed to detect the hypothetical Higgs boson in a single year of data taking at design luminosity. In order to create the detector a collaboration of more than 2000 physicists from 38 countries at 175 institutions has been formed and has worked for more than a decade to design, construct, install, test and commission the detector.

In 2005 and 2006 the detector elements were brought to the above group Assembly Hall and testing began. By the latter half of 2006, major elements of the Muon subsystem and the Hadronic calorimeter were in place, along with small segments of the Electromagnetic Calorimeter and the Tracking system. During 2006, the magnet was fully powered and the field was mapped. In addition, the subsystems were fully time synchronized using a common cosmic ray muon source. This data allowed for a preliminary alignment of the Muon system. In fact, the resulting measurement of the muon charge ratio will soon be published and is shown in Fig. 2.
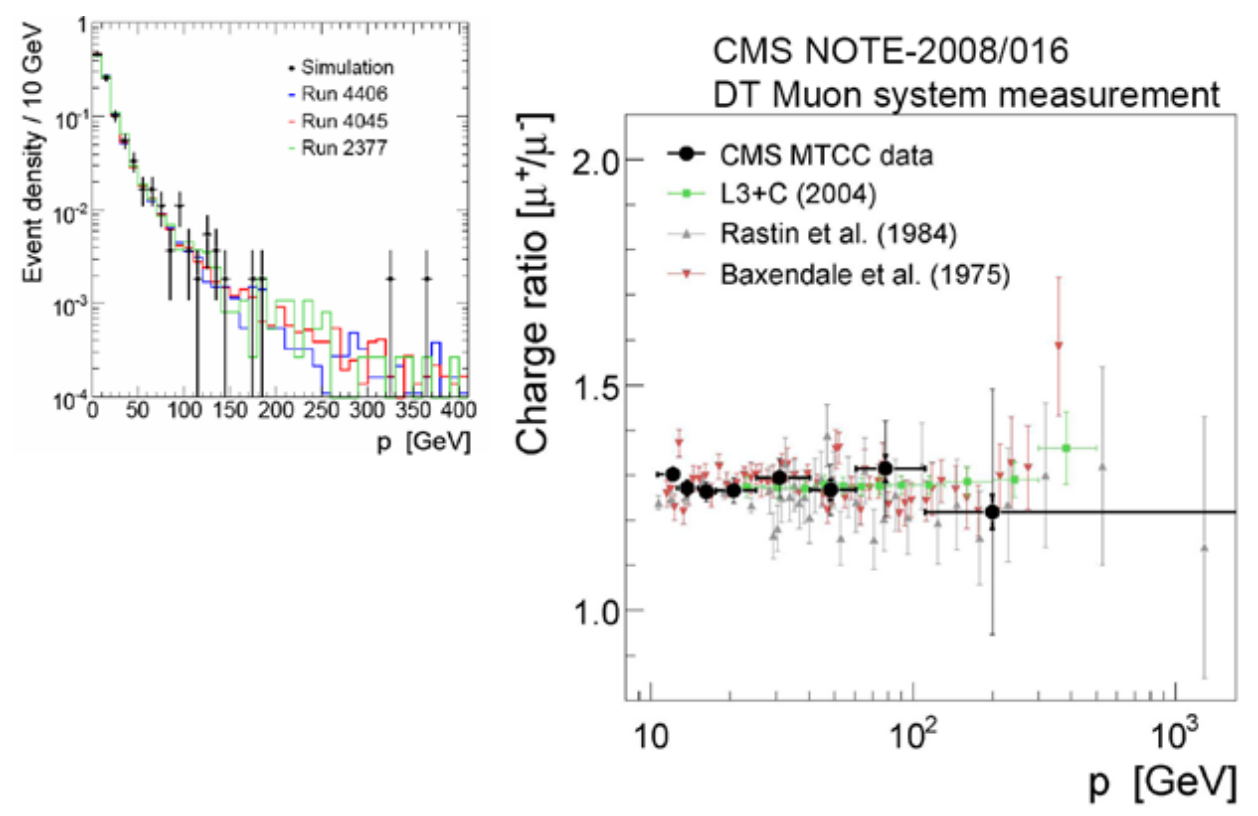

Figure 2: Results from the Assembly Hall tests in late 2006 for the momentum spectrum of the cosmic muons and the charge ratio. Note that the spectrum of cosmic muons extends to hundreds of $\mathrm{GeV}$.

\section{Lowering CMS into the Collision Hall}

After the Assembly Hall tests, elements of the CMS detector were lowered into the Collision Hall in 2007 and 2008. The largest single piece was lowered in February 2007 and consisted of the central magnet yoke and associated muon chambers, along with the CMS magnet coil. A picture of this operation appears in Fig. 3. 


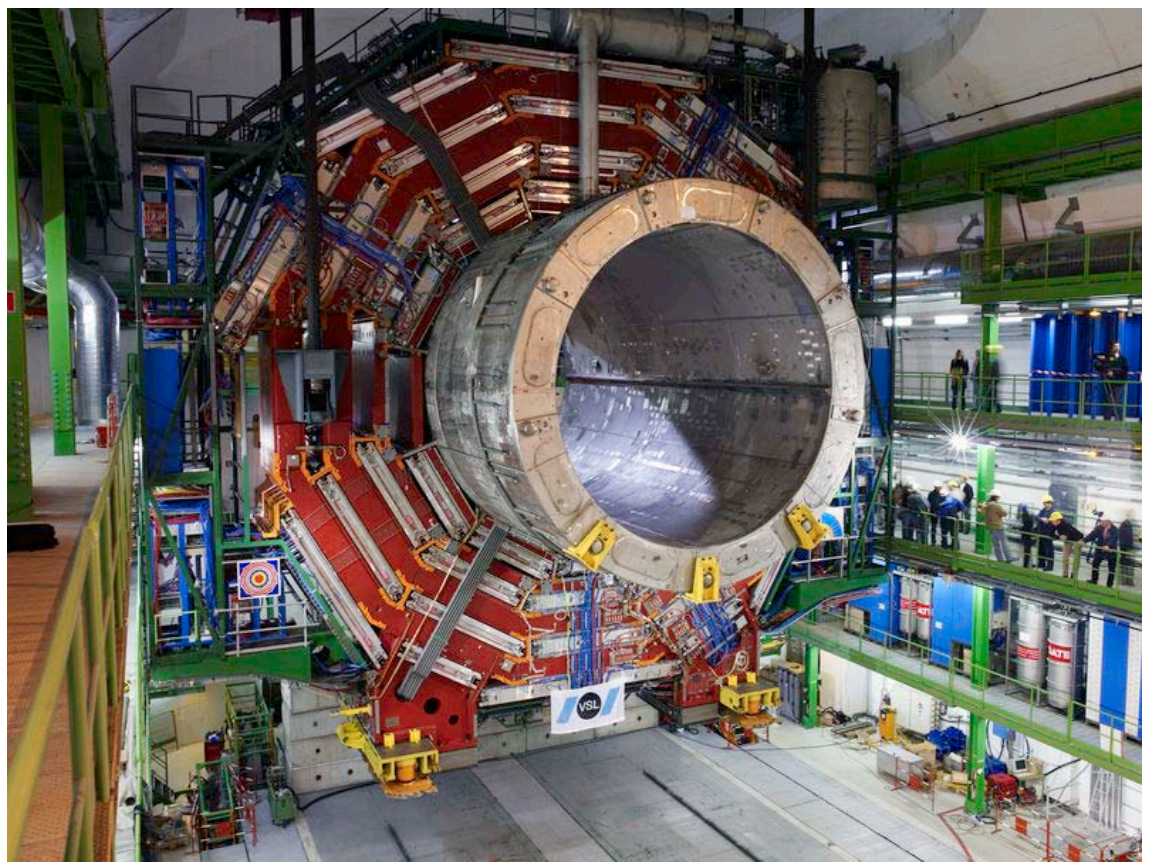

Figure 3: Lowering of the central "wheel" of CMS in early 2007. The return yoke steel and the Muon chambers are clearly seen. The solenoid cryostat is also very evident. The scale can be set by referring to the people seen on the catwalks.

In January 2008, the last of the heavy elements was lowered into the Collision Hall. These were connected to services and cosmic ray data taking commenced. Subsequent elements which were installed participated in the series of cosmic ray runs as they began commissioning. In December 2007, the silicon strip Tracker was installed. After that device was cabled, the beam pipe was installed and baked out in May 2008. The endcap ECAL, EE, followed in the summer of 2008. Finally in August 2008, the pixel Tracker system was installed and connected. Of necessity, this installation had to come after the bake-out of the beam pipe. During most of 2008, CMS was able to continue to take cosmic ray data of increasing scope and complexity as more and more elements of CMS were newly installed.

\section{Cosmic Ray Tests in the Collision Hall}

The final configuration of CMS is shown in Fig. 4 where the detector is completely closed with LHC shielding in place ready for LHC beam. 


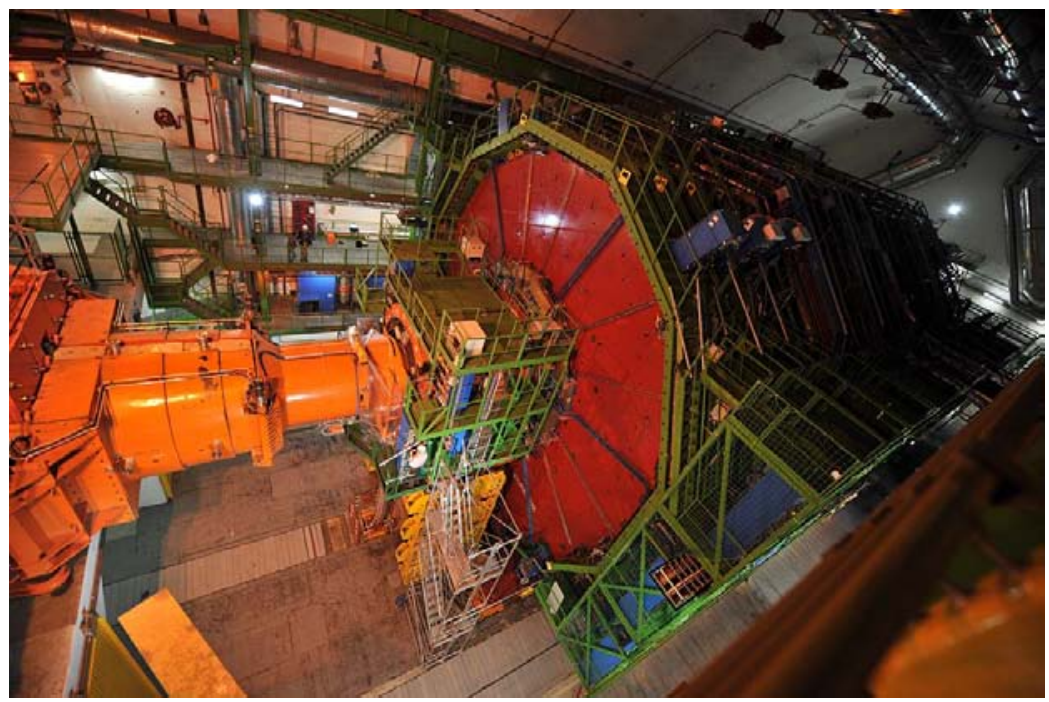

Figure 4: The configuration of CMS in Sept. 2008 when CMS was closed and ready for beam. Note the cylindrical orange shielding on the left which continues the HF calorimetric coverage to smaller angles and shields nearby LHC machine elements.

During this preparatory period, CMS achieved design pre-alignment specifications of the Tracking and Muon systems using momentum analyzed cosmic ray muons. All detector systems were also time synchronized to a few nsec. In addition, the calorimetry used test beam data taken on a subset of their modules to compare to radioactive sources and cosmic ray muons so as to achieve a redundant pre-calibration of the calorimeter energies to 5\%.

CMS was, therefore, well prepared for the first LHC beams in September 2008. An event display showing the full CMS detector is shown for a cosmic ray event in Fig.5. The muon track is seen in the muon system, the EM and hadronic calorimetry and in the silicon tracker. Clearly CMS was ready for the LHC beam.

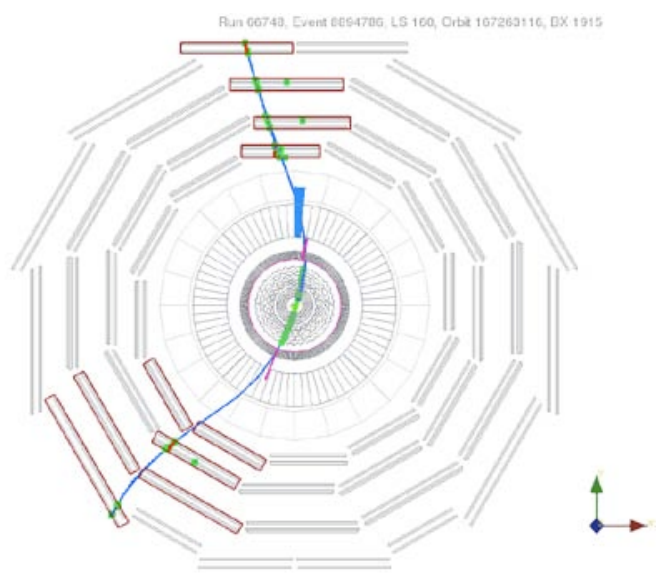

Figure 5: Event display for CMS cosmic ray running taken in late 2008. The cosmic ray track in the Muon system and the Tracker system is quite evident. The muon also deposits minimum energy in the calorimetry which serves as a reference energy for calibration. 


\section{LHC Beam in 2008}

In the fall of 2008, the first beam was injected into the LHC ring at an energy of $0.45 \mathrm{TeV}$. The beams were then brought around the rings and, typically, dumped into collimators upstream of the experiments. These events had $\sim 10^{9}$ protons interacting in the collimators and sent a "wall" of muons toward the CMS detector. In fact, these events essentially fired every element of CMS. Note that the Tracker systems were kept off at this time in order to protect them. A typical event of this type is shown in Fig. 6.

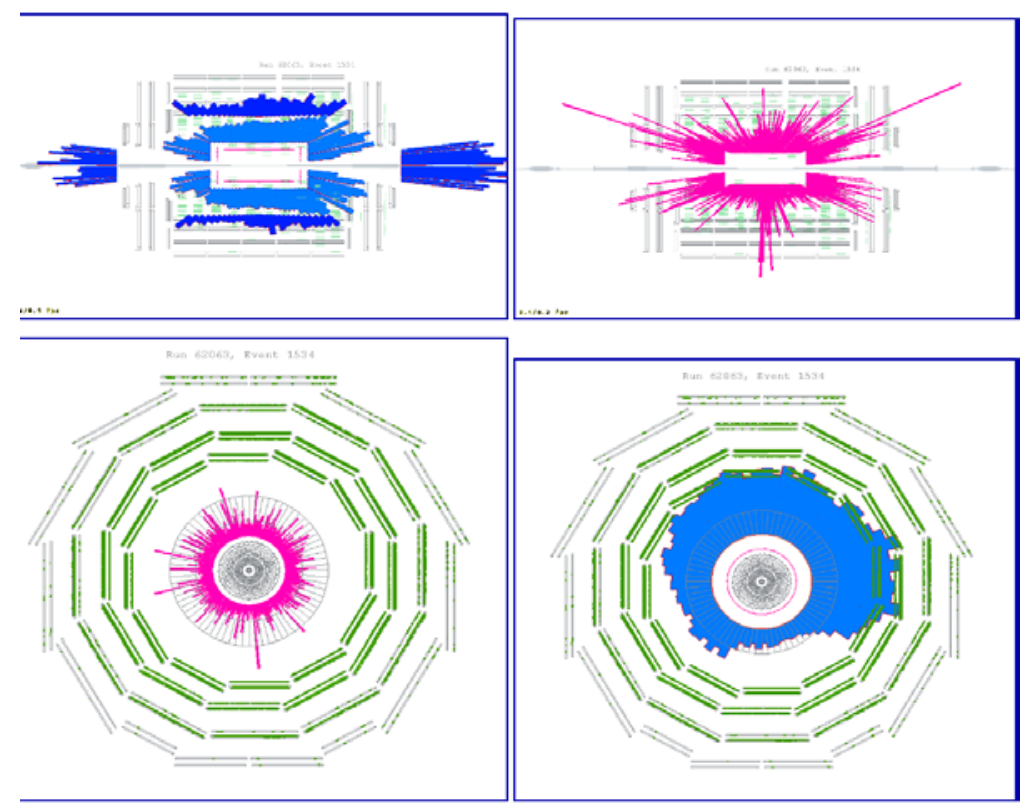

Figure 6: Events with LHC beam at $0.45 \mathrm{TeV}$ dumped into an upstream collimator which is off the right side in these plots. The HCAL is shown in blue with HB, HE and HF all "lit up". The ECAL is in pink with EB and EE filled. The muon chambers, in green, are fully occupied with muon hits.

These events were also used to check the time synchronization of the CMS elements since they are a thin "wall" of muons crossing the detector in time coincidence moving at the velocity of light. A typical time alignment for HCAL is shown in Fig. 6. Clearly, the CMS detector elements can now all be individually timed to a few nsec channel to channel. 


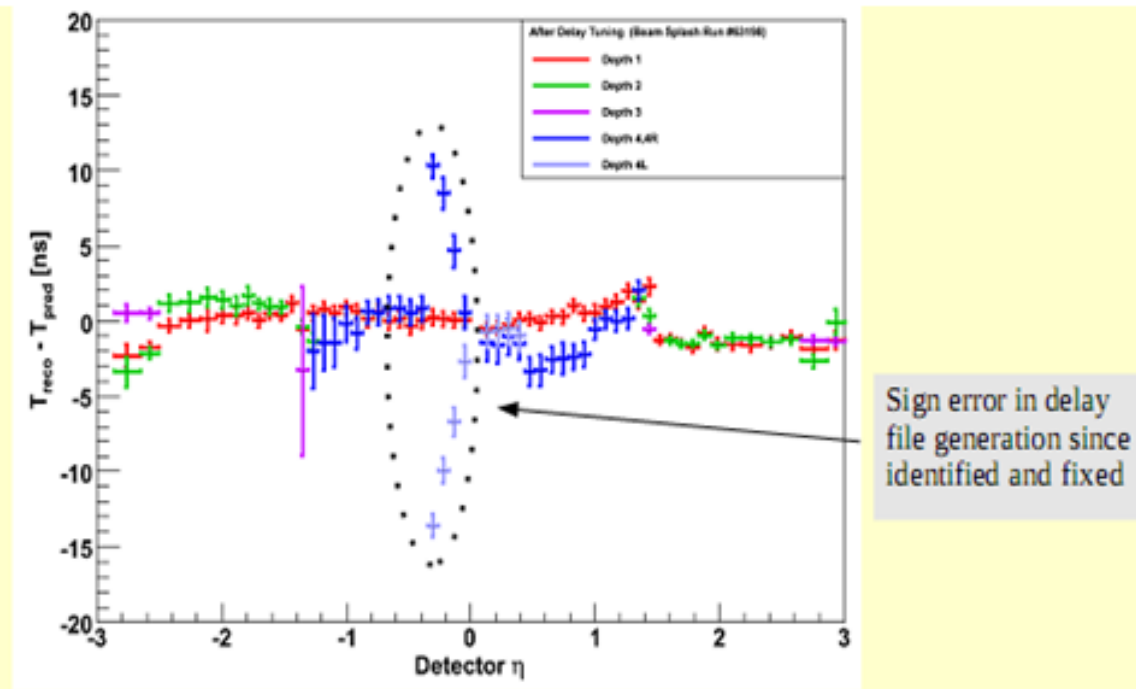

Figure 7: HCAL timing across the full rapidity range of HB and HE using "collimator splash" events.

After dumping the beams, the LHC accelerator experts then managed to r.f. capture the beams for multi-turn operation. In this situation, the CMS detector saw mostly muons in the halo of the beam due to beam scraping. This data allowed CMS to check the reconstruction software for the endcap muon system. A typical event of this type is shown in Fig.8.

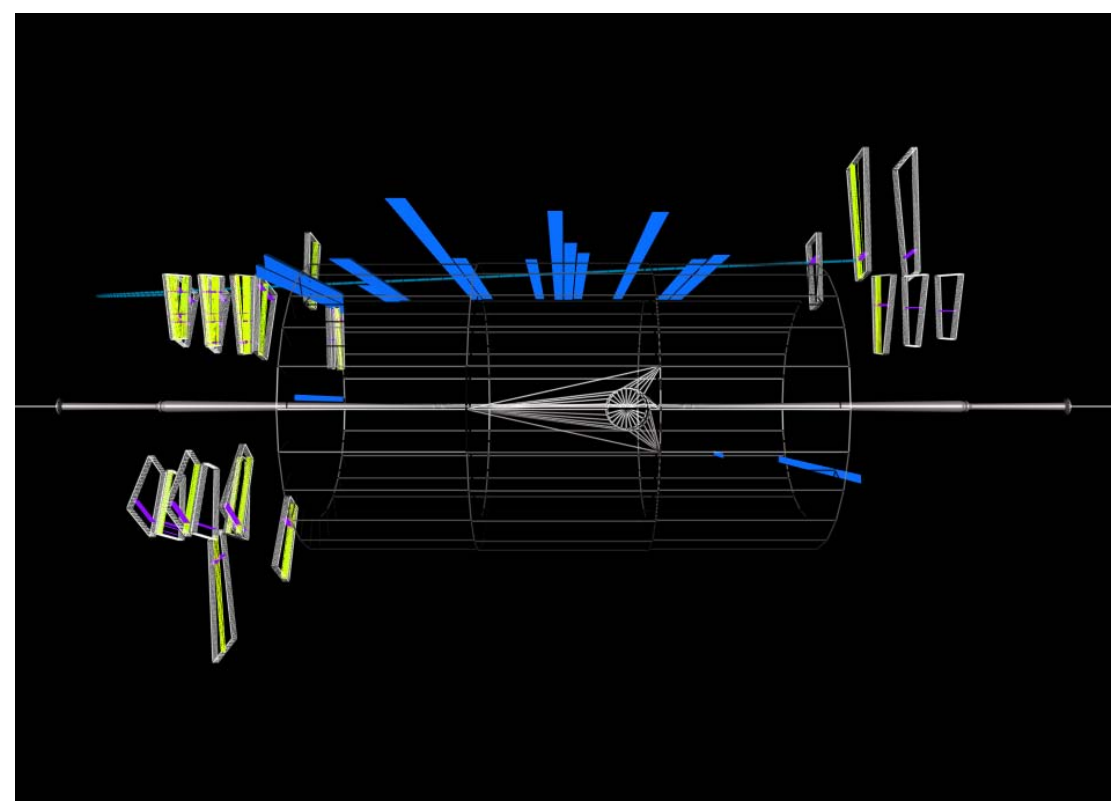

Figure 8: Reconstructed beam halo muons with r.f. captured beam circulating around the LHC ring. Note the hits in both Muon endcap systems and in the HCAL. 
It appears that the captured beam is reasonably well behaved and that the muons from beam scraping can be found and reconstructed fairly easily. This bodes well for the future.

The Muon systems were tuned up using the muon halo for additional alignment checks. The reconstruction package showed that the individual detector planes of CSC in the endcaps were functioning very efficiently, with an average efficiency of $98.9 \%$ to reconstruct a track segment in each of the 4 CSC stations.

After the incident when the LHC accelerator complex was damaged, CMS decided to record an extensive set of cosmic ray events at full magnetic field. These events have, in the 300 million collected, a substantial number with muons above the critical energy in iron. Therefore, events were logged with substantial radiated energy in the CMS calorimetry. These events, where a typical such is shown in Fig. 9, will be used to check the muon system momentum scale against the tracker momentum scale and the calorimetric energy scale. The relative timing will also be checked using these events. In this fashion, CMS will be as prepared as possible for the 2009 data taking period.

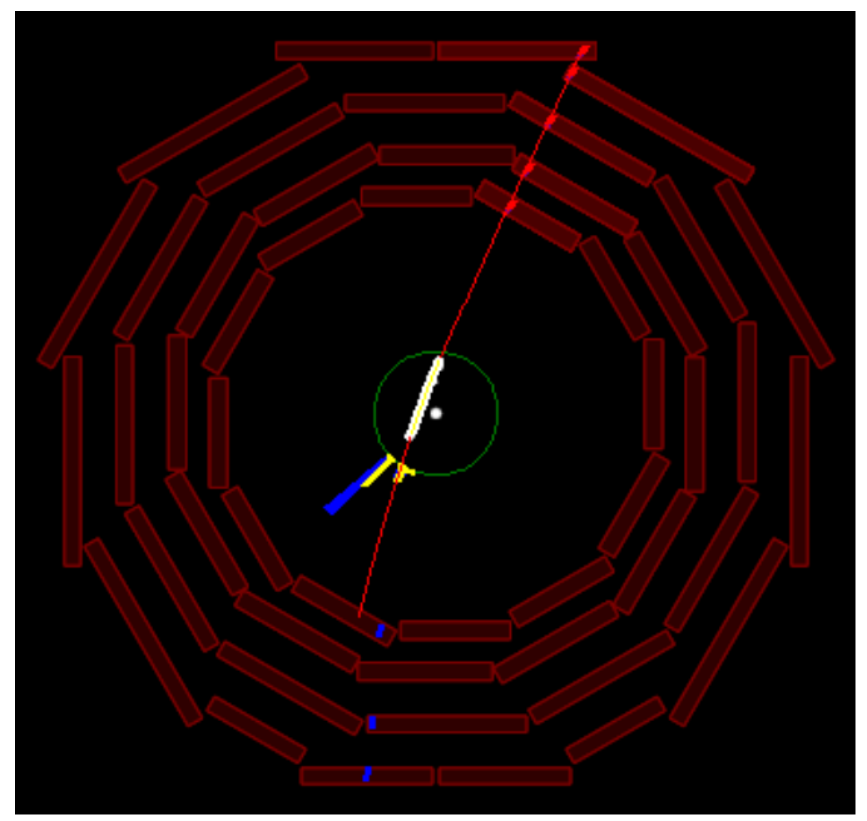

Figure 9: Event logged at CMS where an incoming high energy muon passes through the tracker and radiates into the lower calorimetry, leaving the residual muon detected in the lower muon chambers.

\section{Software and Computing - Data and the Grid}

During the beam tests the trigger system of CMS used only the simplest elements. However, much work has already gone on to commission the Trigger system. Trigger Tables have been defined for early running at low luminosity using the Monte Carlo model of CMS to estimate the trigger rates.

CMS plan to trigger on "minbias” for LHC startup. As luminosity increases jet and lepton triggers will be brought into play. Starting with the cosmic ray running both data quality and trigger monitoring have been put into place. The DAQ system has been run at $\sim 50 \mathrm{kHz}$ and has thus been "stress tested" but in the absence of real data Monte Carlo events are used. 
During data taking the experiment will produce about 15 Million Gigabytes of data each year (about 20 million CDs!), To handle this data volume a hierarchal computing model has been created. Using Monte Carlo model data simulations this computing model too has been tested. Data transfers from T1 -> T2 -> T3 in 2007 at full rate were made in a sustained fashion. The grid system in CMS is now ready as pictured in Fig.10.

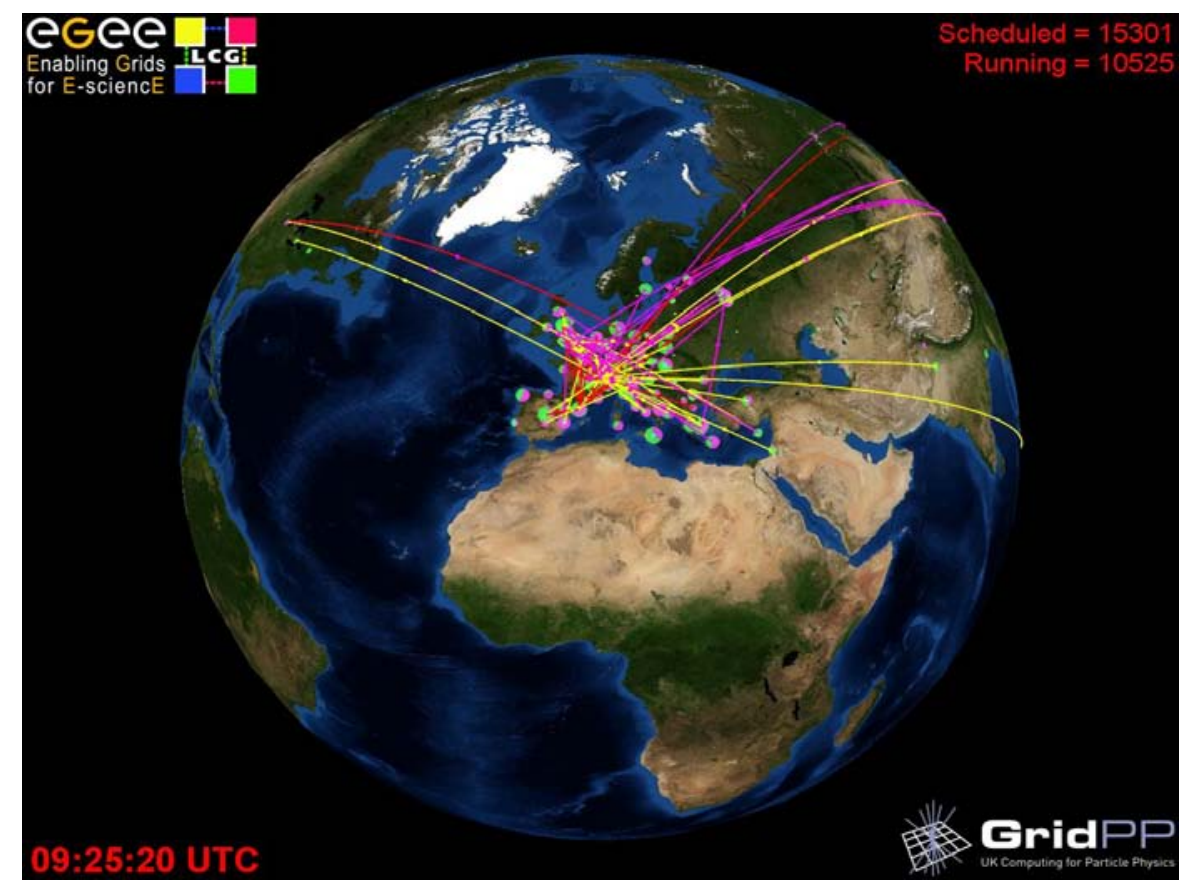

Figure 10: Representation of the data paths for LHC transfers. For CMS the T0 transfer to $7 \mathrm{~T} 1$ and thence to $\sim 50 \mathrm{~T} 2$ sites.

For CMS the CCRC08 test of the entire software and computing system was successful. The tests have demonstrated all key use case performances of T0, CAF, T1, and T2 infrastructure. Some results are; data export by CERN-T1 > 600MB/s, re-reconstruction and skimming run at all T1 sites and physics analysis jobs

successfully run at 62 sites. CMS have also demonstrated successful DPG/ALCA/Physics activities, and at the same time, stress tested the computing infrastructure with real and artificial loads. Thus, the data streaming for alignment and calibration is also in hand.

The LHC is a discovery machine so CMS must be ready on "day one". CMS have practiced data transfer, data quality monitoring and remote data analysis using "global runs". Remote Operations Centers in many sites, for example Fermilab and DESY, mean that more of CMS is engaged. By engaging more of the CMS collaboration CMS plan to get the first physics data analyzed expeditiously.

A picture of the Fermilab remote operations center is shown in Fig. 11. 


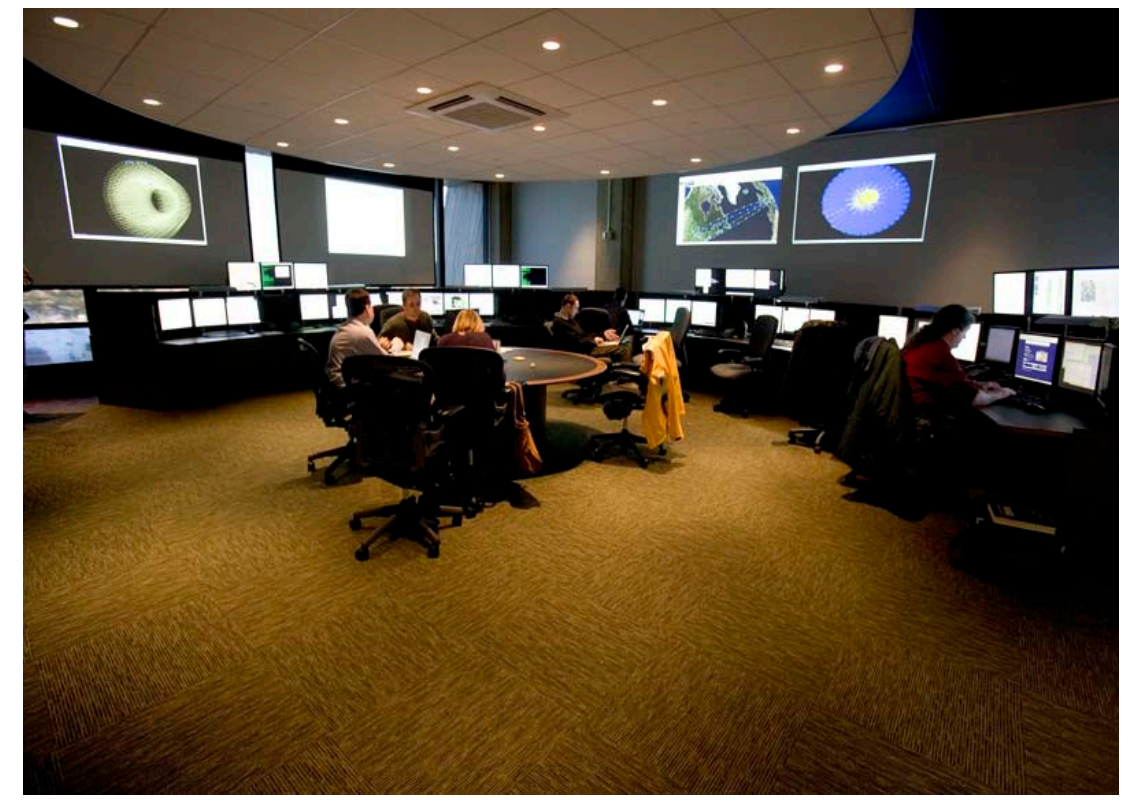

Figure 11: Photo of the Fermilab Remote Operations Center (ROC). This ROC has participated in all of the CMS data taking and data monitoring since the Assembly Hall magnet testing in the fall of 2006

\section{Summary}

- The CMS magnet, MB and HCAL were tested in the Surface Hall in 2006

- After lowering into the Collision Hall all the CMS subsystems were aligned, calibrated and synchronized using cosmic rays.

- $\quad$ LHC beam was successfully used to set timings and confirm reconstruction algorithms.

- Data transfers and analysis models have been exercised

- $\quad$ CMS have taken 300 M cosmic ray events in Oct.-Nov. in preparation for the 2009 run. These data provide additional redundant checks of the detector.

- We are ready for multi-TeV LHC collisions 